\title{
Novel High-Gain Narrowband Waveguide-Fed Filtenna using Genetic Algorithm
}

\author{
Mahdi Ghorbani, Habib Ghorbaninejad \\ Department of Electrical Engineering, University of Guilan
}

\begin{tabular}{|c|c|}
\hline Article Info & ABSTRACT \\
\hline & \multirow{10}{*}{$\begin{array}{l}\text { Filtenna is an antenna with filtering feature. There are many ways to design a } \\
\text { filtenna. In this paper, a high-gain narrowband waveguide-fed aperture } \\
\text { filtenna has been proposed and designed. A patterned plane, which is } \\
\text { designed using genetic algorithm has been used at the open end of the } \\
\text { waveguide fed, mounted on a conducting ground plane. To design the } \\
\text { patterned pattern, magnetic field integral equation of the structure has been } \\
\text { derived, so it has been solved using method of moments. The proposed } \\
\text { filtenna has been simulated with HFSS that confirms the results obtained by } \\
\text { method of moments. Finally, an unprinted dielectric as a superstrate has been } \\
\text { used to enhance the gain of the filtenna. The filtenna bandwidth is } 1.76 \% \\
\text { (160 MHz) which has the gain of } 15.91 \mathrm{~dB} \text { at the central frequency } \\
\text { of } 9.45 \mathrm{GHz} \text {. } \\
\text { Copyright } @ 2017 \text { Institute of Advanced Engineering and Science. } \\
\text { All rights reserved. }\end{array}$} \\
\hline Received Sep 11, 2016 & \\
\hline Revised Nov 27, 2016 & \\
\hline Accepted Dec 18, 2016 & \\
\hline Keyword: & \\
\hline Filtenna & \\
\hline Genetic algorithm & \\
\hline High gain & \\
\hline Method of moment & \\
\hline Narrow band & \\
\hline
\end{tabular}

Corresponding Author:

Habib Ghorbaninejad,

Department of Electrical Engineering,

University of Guilan,

University of Guilan, Rasht, Guilan Province, Iran.

Email: ghorbaninejad@guilan.ac.ir

\section{INTRODUCTION}

In communication systems with sensitive receiver and transporter, it is necessary to filter the unwanted signals. Often this unwanted signals are strong compared to the main signal and therefore it should be eliminated. The best way for eliminating this unwanted signals is filtenna. Filtenna is a multifunction device, which is done filtering feature and radiation at the same time. Therefore the main signal is protected. There are many way for design of various type of filtenna with narrow or wideband application. Also many technics is used for adjust bandpass and rejection band of filtering feature.

Microstrip antenna is one of the most attractive types of antennas. Microstrip antenna usually is used in wideband application, but in some literature it is used in narrowband, or in multiband application. With an umbrella shape radiation element, and modified ground plane, filtering feature can be achieved [1]. Increasing the number of resonant frequency can enhance the bandwidth of the filtenna [2]. In [3] wideband microstrip antenna is used, and narrowband filtering feature is achieved with electronically adjusting of resonant frequency. Also, defected ground plane [4], and H-shape slot on the microstrip [5] can create several resonant frequency. The gain of the antenna is one of the most important factors in the design process, while the mentioned antennas suffer from the deficiency of undesired gain in the frequency range.

Substrate integrated waveguide has also been used for filtenna design. In [6] horn antenna with FSS is used with acceptable gain. Filtering feature can obtained through using coupled resonator, which the last resonator also contains the radiating element [7]. Filtenna with balun and Yagi-Uda antenna as driven elements has been investigated in [8], which have a low gain with complex configuration. Substrate integrated waveguide base antenna is used for filtenna design [9]. 
In [10] filtenna is based on wideband coupled planar waveguide (CPW) antenna, which filtering feature add rejection band to the antenna. This filtenna have a low gain. In [11] DRA multiband filtenna is designed. In this paper three-segmental relatively low permittivity thin dielectric has been used and two metal plates are applied to set four different frequency band. Also, planar phased-array antenna with frequency selectivity properties has been investigated, so that filtering characteristic has been obtained in the commonmode rejection module, consisting in a planar hybridring [12].

In this paper a novel filtenna is proposed. The filtenna is based on waveguide-fed aperture antenna. For filtering feature, simple aperture is replaced by a patterned plane one which is mounted on the ground plane. Green's function of the form of magnetic field integral equations (MFIEs) in the waveguide is derived and solved using method of moments. The desired patterned plane is obtained from genetic algorithm using optimization. At the final step for the gain enhancement, unprinted high permittivity dielectric slabs are used in front of the aperture as superstrate, based on Fabry-Perot theorem. The proposed filtenna has a good gain, return loss at the operating frequency.

\section{DESIGN METHOD OF THE PROPOSED STRUCTURE}

\subsection{The Proposed Filtenna Structure}

The proposed antenna is based on waveguide-fed aperture antenna with patterned plane mounted on a ground plane, along with two unprinted dielectric slabs (as superstrates) which are located over patterned plane for gain improvement. Patterned plane is printed on a dielectric substrate. Figure 1 shows three dimensional view of the proposed filtenna configuration. For this purpose WR90 waveguide with dimension of $22.86 \times 10.16 \mathrm{~mm}$ is selected. WR90 is used in X-band at $8-12 \mathrm{GHz}$. Substrate with low permittivity of 1.2 is selected. Figure 2 shows the side view of the proposed filtenna along with waveguide fed structure.

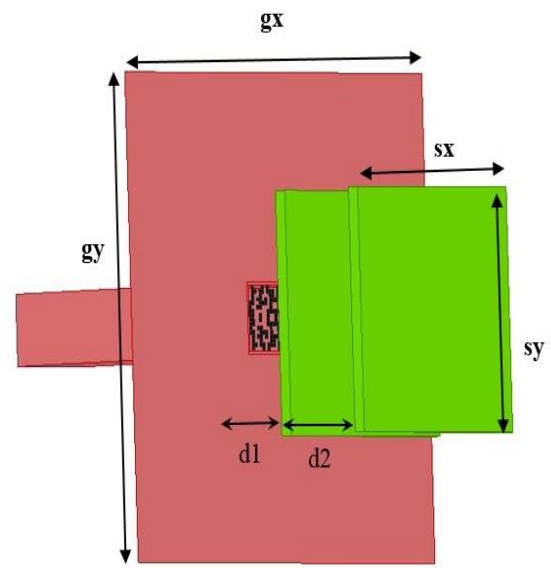

Figure 1. Three Dimensional View of the Proposed Filtenna Configuration

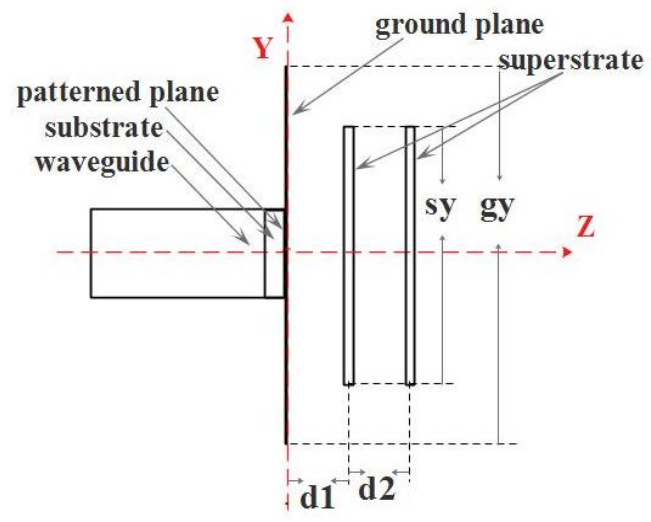

Figure 2. The Side View of the Proposed Filtenna along with Waveguide Fed Structure 


\subsection{Green's Function and Magnetic Field Integral Equations (MFIEs)}

At the first step in the design process, the desired pattern should be obtained. For this purpose, the electric and magnetic field in the various regions, including substrate layer and hollow waveguide section is written. Boundary conditions are applied at the common surface between substrate and air inside of the waveguide, and between common surface between substrate and air out of the waveguide and the following matrix equation is achieved.

$$
\left[\begin{array}{l}
H_{x}^{d} \\
H_{y}^{d}
\end{array}\right]=\left[\begin{array}{ll}
G_{x x} & G_{x y} \\
G_{y x} & G_{y y}
\end{array}\right]\left[\begin{array}{l}
M_{x} \\
M_{y}
\end{array}\right]
$$

where $H_{x}^{d}$ and $H_{y}^{d}$ are the magnetic fields in dielectric substrate in x and y direction respectively. $M_{x}$ and $M_{y}$ are magnetic current in x and y direction respectively. $G_{x x}, G_{x y}, G_{y x}$ and $G_{y y}$ are green functions that are given as the following:

$$
\begin{aligned}
& G_{x x}=-\left(Y^{M} \cos ^{2} \theta+Y^{E} \sin ^{2} \theta\right) \\
& G_{x y}=G_{y x}=\left(Y^{M}-Y^{E}\right) \cos \theta \sin \theta \\
& G_{y y}=-\left(Y^{E} \cos ^{2} \theta+Y^{M} \sin ^{2} \theta\right)
\end{aligned}
$$

where $Y^{M}, Y^{E}, \cos \theta, \sin \theta$ are as follows:

$$
\begin{aligned}
& Y^{M}=Y_{d}^{T M} \frac{Y_{a}^{T M}+Y_{d}^{T M} \tanh \left(\gamma_{d} d\right)}{Y_{d}^{T M}+Y_{a}^{T M} \tanh \left(\gamma_{d} d\right)} \\
& Y^{E}=Y_{d}^{T E} \frac{Y_{a}^{T E}+Y_{d}^{T E} \tanh \left(\gamma_{d} d\right)}{Y_{d}^{T E}+Y_{a}^{T E} \tanh \left(\gamma_{d} d\right)} \\
& \sin \theta=\frac{k_{x}}{\sqrt{k_{x}^{2}+k_{y}^{2}}} \\
& \cos \theta=\frac{k_{y}}{\sqrt{k_{x}^{2}+k_{y}^{2}}}
\end{aligned}
$$

where $Y_{a}^{T M}, Y_{d}^{T M}$ are admittance of air and dielectric substrate in TM mode, respectively, and $Y_{a}^{T E}, Y_{d}^{T E}$ are admittance of air and dielectric substrate in TE mode, respectively. $k_{x}=m \pi / a, k_{y}=n \pi / b$, where a, b are the WR90 dimensions and $\mathrm{m}, \mathrm{n}$, is non-negative integers due to propagating or evanescent modes.

To solve the obtained equations, unknown magnetic current $M_{x}$ and $M_{y}$ on the patterned plane (corresponding to non-metallic parts of patterned plane) in the $\mathrm{x}$ and $\mathrm{y}$ direction is expanded in terms on sub domain basis functions to apply method of moments, as follows:

$$
\begin{aligned}
& M_{x}=\sum_{p=1}^{N_{x}} C_{p}^{x} B_{p}(x) \\
& M_{y}=\sum_{q=1}^{N_{y}} C_{q}^{y} B_{q}(y) \\
& B_{p}(x)=\frac{\sin \left[k_{0}\left(\Delta_{x}-\left|x-x_{p}\right|\right)\right]}{\operatorname{Sin}\left[k_{0} c\right]} \\
& B_{q}(y)=\frac{\operatorname{Sin}\left[k_{0}\left(\Delta_{y}-\left|y-y_{q}\right|\right)\right]}{\operatorname{Sin}\left[k_{0} c\right]}
\end{aligned}
$$

The integral equation of magnetic field is solved using method of moment (Galerkin Method). This result is used in MATLAB in genetic algorithm optimization to find the best pattern, which is match with given specifications. 


\subsection{Genetic Algorithm Optimization}

Genetic algorithm is a useful way for complicated optimization. For example in [13], genetic algorithm has been used in smart antenna for fast dynamically change of the environment. In this paper we have used the genetic algorithm to match the return loss of the patterned plane with desired one.

Waveguide cross section is divided to $24 * 16$ rectangular subsections. For a given patterned plane, some of the subsections are metalized and the remained ones are non-metalized. The aim is to fine a patterned plane with given specifications. Here, patterned plane has been considered to have symmetry in $\mathrm{x}$, y directions to reduce optimization time and computer memory; therefore just one quarter of waveguide cross section, means, $12 * 8$ subsections is used on genetic algorithm optimization. Then desired return loss of the filtenna with that of a simple series LC circuit is matched to meet filtering feature.

\subsection{The Proposed Filtenna Results}

Figure 3 show the designed patterned plane obtained using genetic algorithm. The proposed structure has been simulated with HFSS. Figure 4 show the return loss of the desired resonator compared with that of the designed patterned plane. Resonant frequency of the desired resonator has been assumed 10 $\mathrm{GHz}$, but the designed resonant frequency in HFSS simulation result is equal to $9.77 \mathrm{GHz}$.

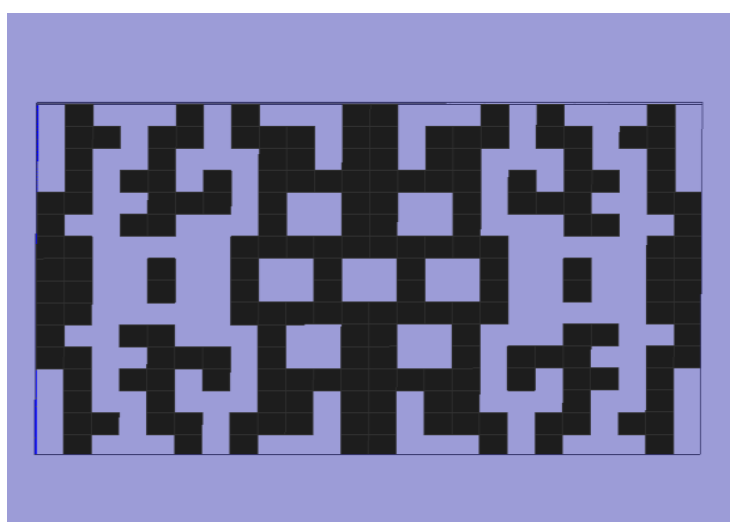

Figure 3. The Patterned Plane Obtained using Genetic Algorithm

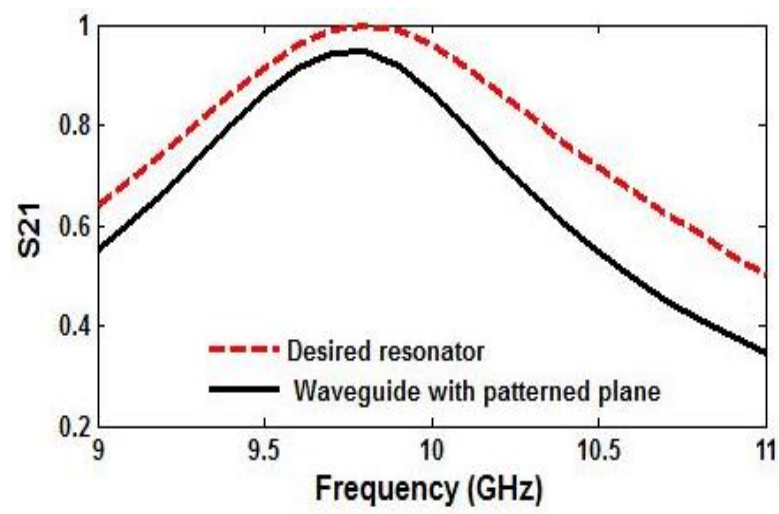

Figure 4. Return loss of Desired Resonator Compared with that of the Designed Patterned Plane

Figure 5a shows the return loss of the filtenna before and after locating of the patterned plane. It shows the effect of the patterned plane. Fig. $5 \mathrm{~b}$ is the gain of the filtenna before and after using patterned plane and it shows that this patterned plane decrease the gain of the filtenna.

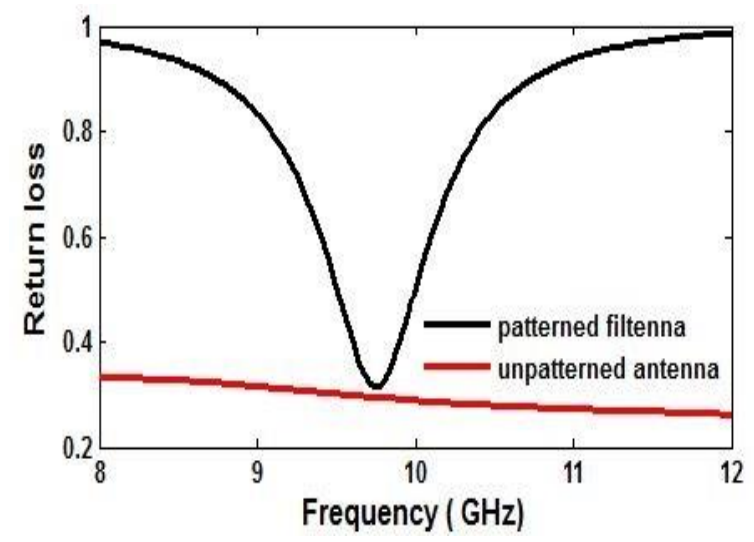

(a)

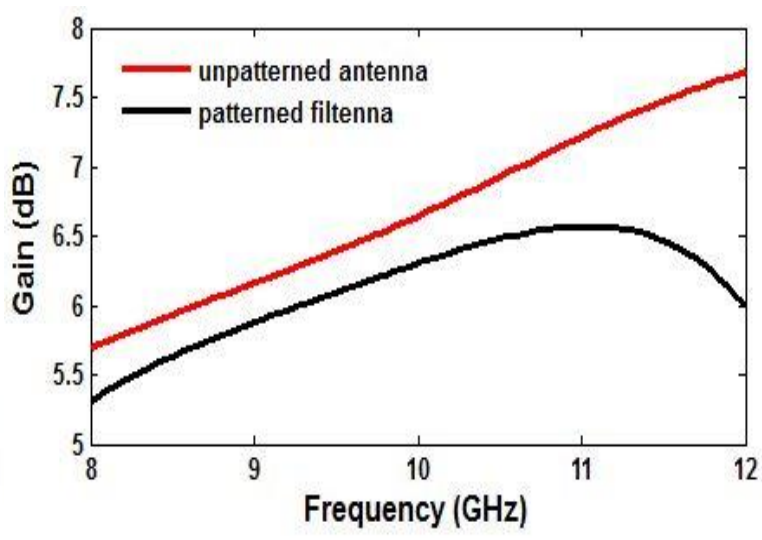

(b)

Figure 5. (a) The return loss and (b) The Gain of the Proposed Filtenna before and after using Patterned Plane 


\subsection{Gain Enhancement}

Using Partially Reflecting Sheet (PRS) as superstrate in front of the aperture and parallel to the ground plane at a suitable distance, it is possible to enhance the gain of this type of antennas. This introduced first time with Trentini in 1956 [14]. This improvement is based on cavity model, which is created between ground plane and superstrate. Beam is multi-reflected between these two planes, and leaked from superstrate to the broadside. The maximum gain is obtained while maximum numbers of these beams are in same direction.

$$
\begin{aligned}
& G=\frac{1+\rho}{1-\rho} \\
& B W=\frac{\Delta f}{f_{0}}=\left(\frac{\lambda}{2 \pi l_{r}}\right) \frac{1-\rho}{\rho^{0.5}} \\
& d_{1}=\left(\frac{\psi_{0}}{360}-0.5\right) \frac{\lambda}{2}+N \frac{\lambda}{2}
\end{aligned}
$$

where $\mathrm{G}$ is the gain, BW is the bandwidth, $d_{1}$ is the distance between ground plane and superstrate, $\rho$ is the reflection coefficient magnitude, $\lambda$ is wavelength, and $\psi_{0}$ is phase angle of the reflection coefficient.

Final configuration of this filtenna is composed of an aperture mounted on the center of conducting ground plane, which is fed with waveguide. Two high permittivity dielectrics as superstrates are located in front of the aperture at the given distance. Superstrates is assumed RO3010 with permittivity of 10.2. Distance between ground plane and superstrate is approximately $d_{1} \cong \lambda / 2$ and $d_{2}=32.6 \mathrm{~mm}$.

\subsection{The structure design parameter}

At this design, WR90 waveguide is used with dimension of $22.86 \times 10.16 \mathrm{~mm}$. The central frequency of the proposed antenna is $9.45 \mathrm{GHz}$, corresponding to wavelength of $31.75 \mathrm{~mm}$. The dimension of each section on the patterned aperture is $0.93 \times 0.65 \mathrm{~mm}$. The aperture located at the center of ground plane with dimension of $\mathrm{Xg}=110 \mathrm{~mm}$ and $\mathrm{Yg}=75 \mathrm{~mm}(3.5 \lambda \times 2.4 \lambda)$. In front of the aperture two layers of superstrates is used. Superstrates are unprinted dielectric and they are chosen RO3010 material with $\varepsilon r 1=10.2$, and they are located at distance of $d_{1}=16.8 \mathrm{~mm}$ and $d_{2}=32.6 \mathrm{~mm}(0.53 \lambda$ and $1.06 \lambda)$ from the aperture. Dimension of both superstrates are $\mathrm{Xs}=55.3 \mathrm{~mm}$ and $\mathrm{Ys}=37.9 \mathrm{~mm}(1.75 \lambda \times 1.2 \lambda)$ with thickness of Ts$=2.0 \mathrm{~mm}$. The patterned plane is printed on a dielectric slab of thickness $0.5 \mathrm{~mm}$. The electric permittivity constant of the substrate is chosen $\varepsilon_{12}=1.2$. The resonant frequency moves toward the lower frequency range when substrate permittivity increases.

\subsection{Final results of the proposed filtenna}

This filtenna is simulated with HFSS and the following results obtained. The central frequency of the proposed filtenna is $9.45 \mathrm{GHz}$ with $1.76 \%$ bandwidth $(160 \mathrm{MHz}$ bandwidth, 9.37-9.54 GHz). Figure 6 shows the return loss and the gain of the proposed filtenna.

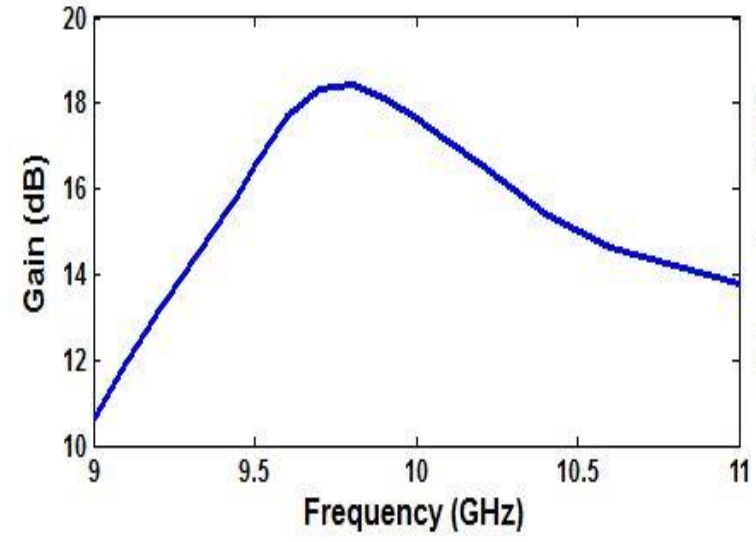

(a)

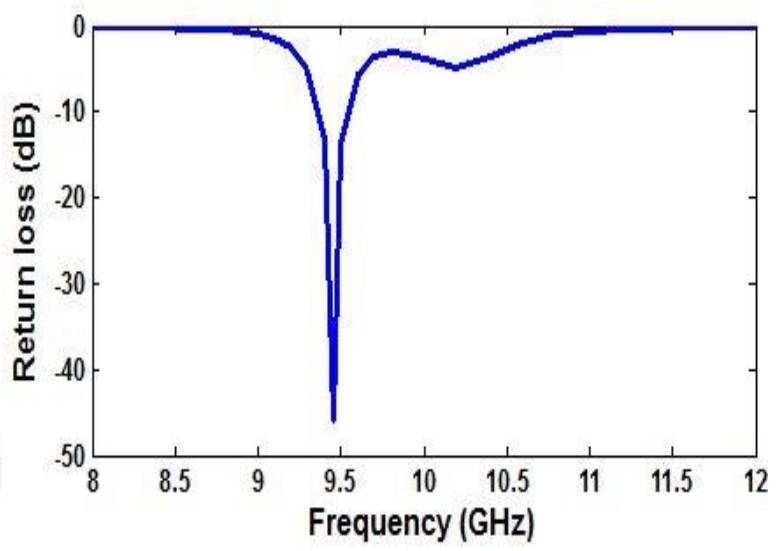

(b)

Figure 6. (a) The return loss and (b) The gain of the proposed filtenn 
It can be observed from Figure 6 that the proposed filtenna gain at the design frequency is $15.91 \mathrm{~dB}$ so that significantly is improved compared with the case without superstrates. Radiation pattern of E-plane and H-plane of the proposed filtenna are shown on Figure 7(a) and (b), respectively. Side lobe level and back lobe level of the proposed filtenna are respectively $15.1 \mathrm{~dB}$ and 19.0dB. Figure 8 shows three-dimensional view of the gain in $\mathrm{dB}$.

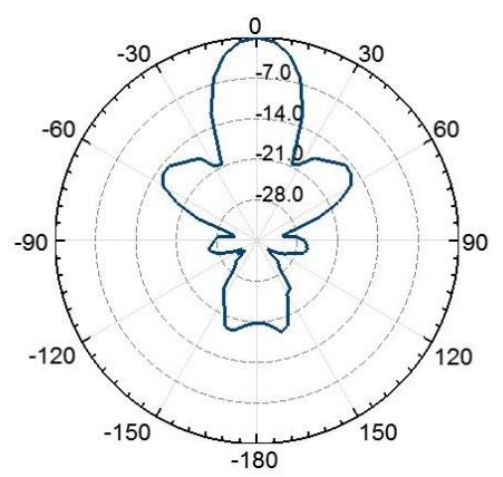

(a)

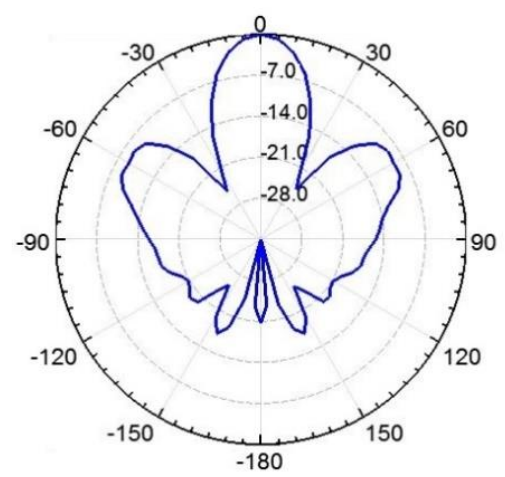

(b)

Figure 7. (a) E-plane and (b) H-plane of the proposed Filtenna

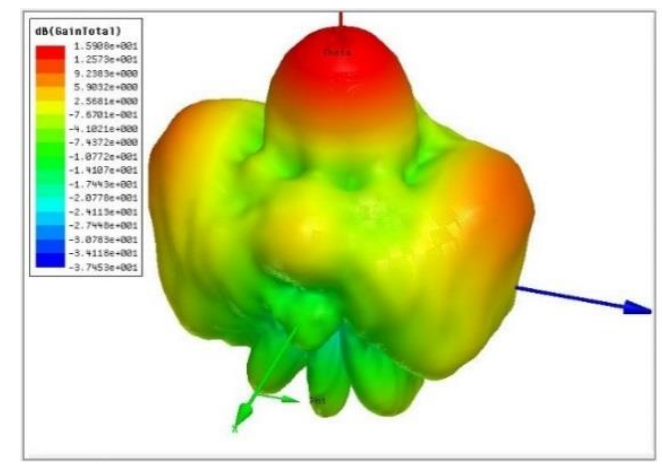

Figure 8. 3-D polar view of the gain in $\mathrm{dB}$

\section{CONCLUSION}

A novel waveguide fed aperture filtenna has been proposed. At this work, magnetic field integral equations solved with galerkin's method to obtain magnetic current density on the patterned aperture. The optimized pattern on printed circuit board (PCB) is obtained using genetic algorithm. Finally the return loss and the gain, has been enhanced using two superstrate and the ground plane.

This filtenna have $9.45 \mathrm{GHz}$ center frequency with $1.76 \%$ bandwidth of $3 \mathrm{~dB}$ and $15.91 \mathrm{~dB}$ gain at the centre frequency. In the proposed filtenna side lobe level and back lobe level are respectively $15.1 \mathrm{~dB}$ and 19.0dB.

\section{REFERENCES}

[1] W. Rongda and G. Peng, "A compact Microstrip Ultra-wideband Filtenna," 15th International conference on electronic packaging technology, pp. 1353 - 1355, 2014.

[2] H. H. M. Ghouz, "New Compact Microstrip Patch Filtenna Structures with Partitioned Ground for 3G/4G Applications," International Journal of Engineering \& Technology IJET-IJENS, vol/issue: 12(05), 2012.

[3] A. H. Ramadan, et al., "A Narrowband Frequency-Tunable Antenna for Cognitive Radio Applications," 6th European Conference on Antennas and Propagation (EUCAP), pp. 3273 - 3277, 2012.

[4] A. Zamanifekri, et al., "Dual-Frequency Filtenna Using Defected Ground Structure for VSAT Applications," Antennas and Propagation Society International Symposium (APSURSI), pp. 434 - 435, 2014. 
[5] R. H. Zaghloul and H. H. M. Ghouz, "A New Compact Multi Resonance H-Patch Filtenna," Antennas and Propagation Society International Symposium (APSURSI), pp. 1718 - 1719, 2013.

[6] G. Q. Luo, et al., "Filtenna Consisting of Horn Antenna and Substrate Integrated Waveguide Cavity FSS," IEEE Transactions on Antennas and Propagation, vol/issue: 55(1), 2007.

[7] O. A. Nova, et al., "Filter-Antenna Module Using Substrate Integrated Waveguide Cavities," IEEE Antennas and Wireless Propagation Letters, vol. 10, pp. 59-62, 2011.

[8] S. Yu, et al., "Integrated Millimeter Wave Filtenna for Q-LINKPAN Application," 6th European Conference on Antennas and Propagation (EUCAP), pp. 1333 - 1336, 2012.

[9] C. Yu, et al., "Ku-Band Linearly Polarized Omnidirectional Planar Filtenna," IEEE Antennasand Wireless Propagation Letters, vol. 11, 2012.

[10] Y. Jang, et al., "Compact Band-selective Filter and Antenna for UWB Application," PIERS Online, vol/issue: 3(7), 2007.

[11] H. Younesiraad, et al., "Small Multi-Band Rectangular Dielectric Resonator Antennas for Personal Communication Devices," International Journal of Electrical and Computer Engineering (IJECE), vol/issue: 4(1), pp. 1 6, 2014.

[12] L. Cifola, et al., "Compact Design of a Planar Filtering Antenna Array including a Frequency Selective CommonMode Rejection Module," Antennas and Propagation Society International Symposium (APSURSI), pp. 1-2, 2012.

[13] J. R. Mohammad, "Comparative Performance Investigations of Stochastic and Genetic Algorithms Under Fast Dynamically Changing Environment in Smart Antennas," International Journal of Electrical and Computer Engineering (IJECE), vol/issue: 2(1), pp. 98 105, 2012.

[14] G. V. Trentini, "Partially reflecting sheet array," IRE Trans. Antennas Propagat., vol. 4, pp. 666-671, 1956.

\section{BIOGRAPHIES OF AUTHORS}

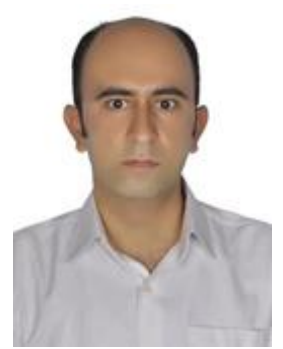

M. Ghorbani received B.Sc. degree in Electrical Engineering from Isfahan University of Technology (IUT), Isfahan, Iran in 2003. He work 10 years in industry as a design and R\&D manager. He received M.Sc. degree in 2016 in Fields and Wave Communication Engineering from University of Guilan.

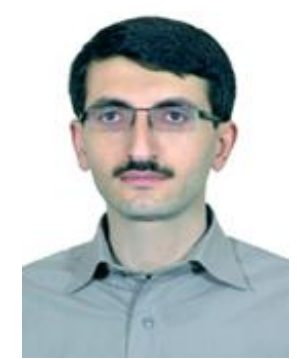

H. Ghorbaninejad received B. Sc. degree from University of Guilan in 2003 and M. Sc. and Ph. D. degrees from Iran University of Science and Technology (IUST) in 2005 and 2010 respectively, all in Fields and Wave Communication Engineering. He is currently assistant professor at Department of Electrical Engineering of University of Guilan. His scientific fields of interest are electromagnetic problems including microwave filter design, compact microwave devices and Green's function of microwave structures. 